\title{
On-pump beating heart treatment in pulmonary embolism and thrombus in transit
}

\author{
Tratamiento del tromboembolismo pulmonar y del trombo en tránsito con circulación \\ extracorpórea sin clampeo
}

\author{
Manuel Roque-Cervetti*, Mariano Camporrotondo, Miguel A. Chiappe, Mariano Vrancic, \\ Fernando Piccinini, and Daniel Navia \\ Department of Cardiac Surgery. Instituto Cardiovascular de Buenos Aires, Buenos Aires, Argentina
}

Thrombi in transit (TT) in right cavities are an infrequent event and it is a medical emergency with very high mortality rate. Without treatment, mortality rate is about $90-100 \%$. Right heart thrombi occur in about $4 \%$ of pulmonary embolism (PE) 1 .

Three types of right heart thrombi can be distinguished by echocardiography: type $A$ is the most common one; it is usually the result of deep venous thrombosis and it has the highest risk of embolization. Type $B$ thrombus is thought to originate within the atrium or ventricle, and it is firmly attached to the chamber wall and it frequently has an ovoid shape. Finally, type $\mathrm{C}$ thrombus is rare and highly mobile. It often resembles cardiac myxomas ${ }^{2}$.

Treatment modalities include anticoagulation therapy, systemic thrombolysis, percutaneous or surgical embolectomy ${ }^{3}$. In other words, optimal therapeutic approach is still a subject of discussion. We present a patient with a type $A$ thrombus treated through a surgical approach.

\section{Case report}

A 47-year-old man was admitted for a syncopal episode and dyspnea. He was hemodynamically stable and eupneic with $\mathrm{O}_{2}$ saturation $94 \%$. As regards his cardiovascular history he referred: deep vein thrombosis and two episodes of pulmonary thromboembolism (PE) in the postoperative context of a hernia disc. Moreover, he completed 1 year of anticoagulation in 2003 which was subsequently suspended. We would like to highlight the mutation of Factor $\mathrm{V}$ Leiden as a relevant antecedent.

The transthoracic echocardiogram (ETT) reported a left ventricle (LV) of normal dimensions, with predominantly septal hypertrophy and preserved systolic function (ejection fraction of $64 \%$ ). An Abnormal movement of interventricular septum secondary to right ventricle (RV) pressure overload was observed. Both the left cavities and the heart valves were normal. At the level of the right cavities we identified dilatation of RA (right atrium) and RV with mild deterioration of the systolic function of the RV: TAPSE $14 \mathrm{~mm}$ and DTI (Doppler tissue imaging) of the tricuspid annulus with $S$ wave of $7 \mathrm{~cm} / \mathrm{s}$. He presented mild tricuspid insufficiency that allowed us to estimate a pulmonary hypertension of $90 \mathrm{mmHg}$. Finally, a homogeneous isoechoic mass with irregular borders, with great mobility was observed in the RA, which extended from the inferior vena cava with direction towards the tricuspid valve and protruded into diastole towards the RV. The image was interpreted as a thrombus in transit in right cavities (Fig. 1).
Correspondence:

${ }^{*} \mathrm{M}$. Roque-Cervetti

E-mail: manuelcervetti_1@ hotmail.com

Available online: $30-01-2020$

Date of acceptance: 23-09-2019 DOI: 10.24875/ACME.M20000099
Arch Cardiol Mex (Eng). 2020;90(1):93-95
www.archivoscardiologia.com

Arch Cardiol Mex (Eng). 2020;90(1):93-95
www.archivoscardiologia.com an open access article under the CC BY-NC-ND 
His angiotomography of pulmonary arteries evidences multiple central filling defects which compromise the main lobar and segmental branches with predominance of the middle lobe and lobar superior right, compatible with acute thromboembolism (Fig. 2).

The patient was surgically treated with a full sternotomy. Aortic arterial and bicaval venous cannulation were performed. Assistance with extracorporeal circulation without clamping was initiated. Right atriotomy and resection of right atrial thrombus were performed. The patient also underwent right pulmonary arteriotomy and thrombectomy. The Pulmonary artery was closed with polypropylene 6-0 and bovine pericardium patch (Fig. 3).

The patient evolved without any complications and he was discharged on the sixth post-surgical day with enoxaparin.

During the initial stage we prefer low-molecular-weight heparins due to several advantages: adequate bioavailability, dose prediction, subcutaneous application. It allows us to use it without being monitored and with a low risk of thrombocytopenia.

One month after surgery we found normal pulmonary pressures (pulmonary artery systolic pressure: $23 \mathrm{mmHg}$ ) in the postoperative control. Currently, he is being periodically checked in hematology.

\section{Discussion}

The treatment of TT is still controversial and difficult. Due to the complexity of the patients and the lack of randomized studies, we continue debating the best therapy for our patients. Different therapeutic approaches for acute PE with concomitant TT have been reported such as anticoagulation with unfractionated heparin (UFH), systemic thrombolysis with recombinant tissue plasminogen activator (rTPA), surgical embolectomy with exploration of the right chambers, pulmonary arteries under full cardiopulmonary bypass, and endovascular thrombectomy ${ }^{1}$.

Pulmonary thromboembolism was present in $98 \%$ of the heart thromboembolism cases analyzed by Rose et al. The treatments administered were none in $9 \%$, anticoagulation therapy in $35.0 \%$, surgical procedure in $35.6 \%$, or thrombolytic therapy in $19.8 \%$. The overall mortality rate was $27.1 \%$. The mortality rate associated with no therapy, anticoagulation therapy, surgical embolectomy, and thrombolysis was $100.0 \%, 28.6 \%$, $23.8 \%$, and $11.3 \%$, respectively ${ }^{4}$.

On the other hand, Athappan et al. studied 328 patients with right heart thrombi (RHT) and PE in a

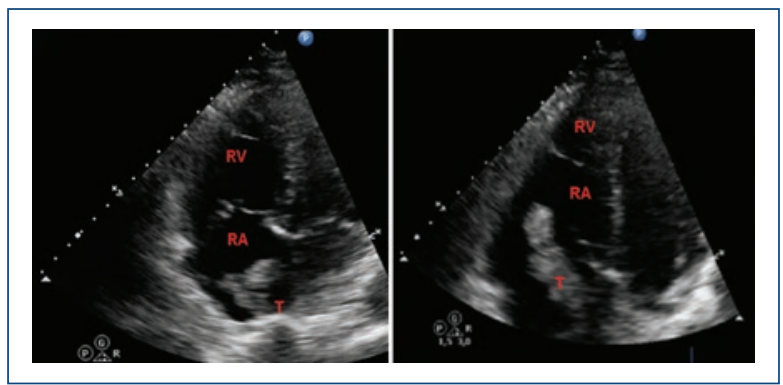

Figure 1. Transthoracic echocardiogram: RV: right ventricle; RA: right atrium; T: thrombus in transit.

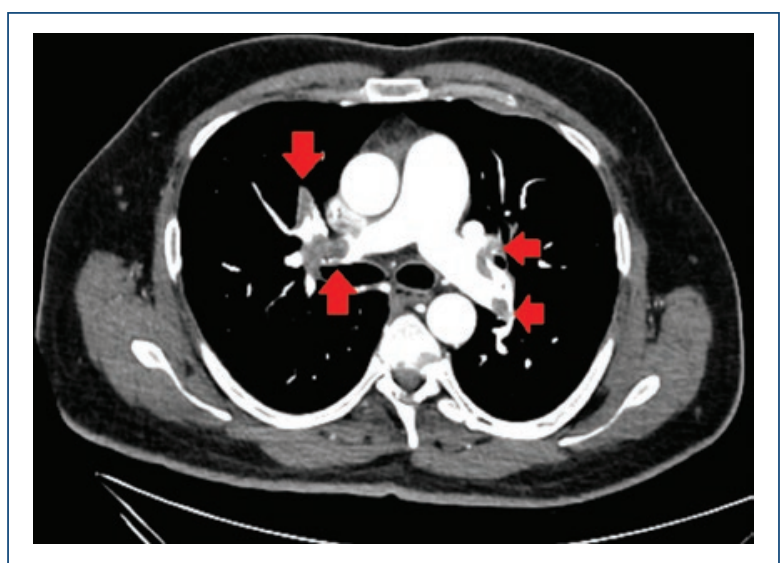

Figure 2. Angiotomography of pulmonary arteries evidences acute thromboembolism.

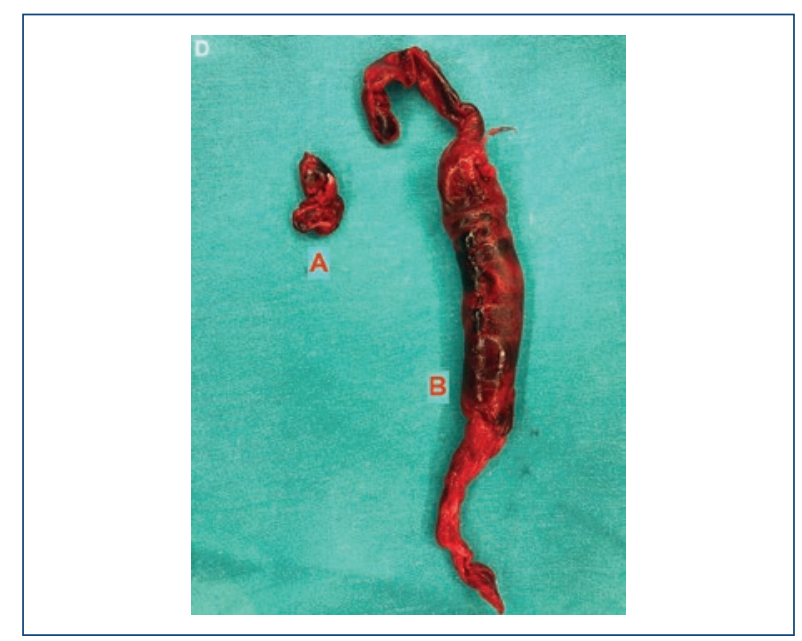

Figure 3. A. Thrombus of right pulmonary artery. B. Thrombus in transit.

meta-analysis. The treatments administered were none in 11 patients (3.4\%), anticoagulation (AC) with heparin in 70 patients $(21.3 \%)$, thrombolytic in 122 patients 
$(37.2 \%)$, catheter-related treatments in five patients $(1.5 \%)$ and surgical embolectomy in 120 patients (36.6\%). The overall short-term mortality for the entire cohort was $23.2 \%$. The mortality rate associated with no therapy was the highest at $90.9 \%$. The mortality associated with AC alone was significantly higher than surgical embolectomy or thrombolysis ( $37.1 \%$ vs. $18.3 \%$ vs. $13.7 \%$, respectively) $)^{5}$.

To conclude, Galeano-Valle et al ${ }^{1}$. reported the surgical management of thrombus-in-transit and pulmonary embolism (PE) in four patients treated with early surgical embolectomy and anticoagulation. The initial treatment was unfractionated heparin (UFH) and urgent right atriotomy and manual removal of the thrombi. All patients survived after 30 days of follow-up.

In our clinical case, the surgical intervention decision was based on: the low pre-operative risk (EuroScore II calculated of $2.56 \%$ and EuroScore Logística of $9.91 \%$ ) and the fact that our surgical team has experience in pulmonary thrombectomy. The patient is being monitored by clinical cardiology and hematology with a life-long anticoagulation based on his history of recurrent episodes of pulmonary thromboembolism and the mutation of factor $\mathrm{V}$ Leiden.

We emphasize the usefulness of the assistance with extracorporeal circulation without clamping, considering that it facilitates the procedure and minimizes risks.

Aortic clamping is not necessary and can be dangerous in elderly patients with calcified aortas (risk of stroke).

Although the evidence tilts the balance towards fibrinolysis and surgery as treatments with better results; we believe it is necessary to individualize the approach depending on the characteristics of the patients and the thrombi. To decide the appropriate treatment for each patient, it is essential to consider the following: the type of thrombus (Type A, B or C), pre-existing pulmonary diseases, deep vein thrombosis, hemodynamic state, experience of the treating team and availability of endovascular treatment ${ }^{6}$.

\section{Funding}

Instituto cardiovascular de Buenos Aires.

\section{Conflicts of interest}

Los autores no presentan conflictos de intereses.

\section{Ethical responsibilities}

Protection of human and animal subjects. The authors declare that no experiments were performed on humans or animals for this study.

Confidentiality of data. The authors declare that they have followed the protocols of their work center on the publication of patient data.

Right to privacy and informed consent. The authors declare that no patient data appear in this article.

\section{References}

1. Galeano-Valle F, Demelo-Rodríguez $P$, García-Fernández-Bravo I, Rodríguez-Abella H, Irabien-Ortiz A, Fortuny-Ribas R, Del Toro-Cervera J. Early surgical treatment in patients with pulmonary embolism and thrombus-in-transit. J Thorac Dis 2018;10(4):2338-2345. doi: 10.21037/ jtd.2018.04.41.

2. European Working Group on Echocardiography. The European cooperative study on the clinical significance of right heart thrombi. Eur Heart $\mathrm{J}$ 1989;10(12):1046-1059.

3. Otoupalova E, Dalal B, Renard B. Right heart thrombus in transit: a series of two cases. Crit Ultrasound J 2017:9:14.

4. Rose PS, Punjabi NM, Pearse DB. Treatment of right heart thromboemboli. Chest 2002;121:806-814.

5. Athappan G, Sengodan P, Chacko P, Gandhi S. Comparative efficacy of different modalities for treatment of right heart thrombi in transit: a pooled analysis. Vasc Med 2015;20(2):131-138.

6. Burgos LM, Mandó F, Furmento JF, Costabel JP. Thrombus in right atrium transit as a finding in a patient with cardiogenic shock. Case report and literature review. Rev Fed Arg Cardiol 2017;46(2):88-90. 